\title{
Recursive General Secret Sharing Scheme Based on Authorized Subsets
}

\author{
Utako Itoh and Kouya Tochikubo
}

\begin{abstract}
The basic idea of secret sharing is that a dealer distributes a piece of information about a secret to each participant in such a way that authorized subsets of participants can reconstruct the secret but unauthorized subsets of participants cannot determine the secret. We propose a new secret sharing scheme realizing general access structures, which is based on authorized subsets. The proposed scheme is perfect and can reduce the number of shares distributed to one specified participant. In the implementation of secret sharing schemes for general access structures, an important issue is the number of shares distributed to each participant. We can apply the proposed scheme to the same access structure recursively. That is, the proposed scheme can reduce the number of shares distributed to another participant once again by applying the proposed scheme recursively. We apply the proposed scheme to all access structures on five participants in order to evaluate the efficiency of the proposed scheme.
\end{abstract}

Index Terms-Secret sharing scheme, general access structure, $(k, n)$-threshold scheme.

\section{INTRODUCTION}

In 1979, Blakley and Shamir independently introduced the concept of secret sharing [1], [2]. In Shamir's $(k, n)$-threshold scheme [1], every group of $k$ participants can recover the secret $K$, but no group of less than $k$ participants can get any information about the secret from their shares. The collection of all authorized subsets of participants is called the access structure. A $(k, n)$-threshold scheme can only realize particular access structures that contain all subsets of $k$ or more participants.

Secret sharing schemes realizing more general access structures than that of a threshold scheme were studied by numerous authors. Koyama proposed secret sharing schemes for multi-groups [3]. Simmons studied secret sharing schemes realizing multilevel access structures [4], [5]. Subsequently, Tassa proposed a hierarchical threshold scheme using polynomial derivatives [6]. Farrás and Padró formalized the concept of hierarchical access structure [7]. Secret sharing schemes based on graph access structures were also proposed [8]-[10]. These schemes obtain the optimal information rates for some access structures, but these schemes cannot be applied to many access structures.

On the other hand, Ito, Saito and Nishizeki proposed a secret sharing scheme for general access structures and showed an explicit share assignment algorithm for any access

Manuscript received June 13, 2018; revised October 15, 2018. This work was supported by JSPS KAKENHI Grant Number 15K00192.

U. Itoh and K. Tochikubo are with the Department of Mathematical Information Engineering, Graduate School of Industrial Technology, Nihon University, Narashino-shi, Chiba 275-8575, Japan (e-mail: ciut16001@g.nihon-u.ac.jp, tochikubo.kouya@nihon-u.ac.jp). structure [11]. Their scheme can realize an arbitrary access structure by assigning one or more shares to each participant. Benaloh and Leichter proposed a secret sharing scheme for general access structures based on a monotone-circuit [12]. Secret sharing schemes which have an explicit assignment algorithm for any access structure are categorized by two types. One type is schemes based on unauthorized subsets [11], [13], [14]. Another type is schemes based on authorized subsets [12], [15], [16].

In the implementation of secret sharing schemes for general access structures, an important issue is the number of shares distributed to each participant. Obviously, a scheme constructed of small shares is desirable. However, in general, the proposed secret sharing schemes for general access structures are impractical in this respect when the size of the access structure is very large.

In this paper, we modify Benaloh and Leichter's scheme [12] and propose a new secret sharing scheme realizing general access structures, which is based on authorized subsets. The proposed scheme is perfect and can reduce the number of shares distributed to one specified participant. We can apply the proposed scheme to the same access structure recursively. That is, the proposed scheme can reduce the number of shares distributed to another participant once again by applying the proposed scheme recursively. We show that the proposed scheme is more efficient than or equal to Benaloh and Leichter's scheme [12] for any access structure. Furthermore, we show that the proposed scheme is more efficient than or equal to Ito, Saito and Nishizeki's scheme [11] for all 180 access structures on five participants.

\section{PRELIMINARIES}

\section{A. Secret Sharing Scheme}

Let $\mathcal{P}=\left\{P_{1}, P_{2}, \ldots, P_{n}\right\}$ be a set of $n$ participants. Let $D(\notin \mathcal{P})$ denote a dealer who selects a secret and distributes a share to each participant. Let $\mathcal{K}$ and $\mathcal{S}$ denote a secret set and a share set, respectively. For sets $A$ and $B$, we denote a difference set by $A-B$. The access structure $\Gamma\left(\subset 2^{\mathcal{P}}\right)$ is the family of subsets of $\mathcal{P}$ which contains the sets of participants qualified to recover the secret. For any authorized subset $A \in \Gamma$, any superset of $A$ is also an authorized subset. Hence, the access structure should satisfy the monotone property:

$$
A \in \Gamma, A \subset A^{\prime} \subset \mathcal{P} \Rightarrow A^{\prime} \in \Gamma \text {. }
$$

Let $\Gamma_{0}$ be a family of the minimal sets in $\Gamma$, called the minimal access structure. $\Gamma_{0}$ is denoted by

$$
\Gamma_{0}=\left\{A \in \Gamma: A^{\prime} \not \subset A \text { for all } A^{\prime} \in \Gamma-\{A\}\right\} .
$$


For any access structure $\Gamma$, there is a family of sets $\bar{\Gamma}=2^{\mathcal{P}}-\Gamma . \bar{\Gamma}$ contains the sets of participants unqualified to recover the secret. The family of maximal sets in $\bar{\Gamma}$ is denoted by $\bar{\Gamma}_{1}$. That is,

$$
\bar{\Gamma}_{1}=\left\{B \in \bar{\Gamma}: B \not \subset B^{\prime} \text { for all } B^{\prime} \in \bar{\Gamma}-\{B\}\right\} .
$$

Let $p_{\mathcal{K}}$ be a probability distribution on $\mathcal{K}$. Let $p_{\mathcal{S}(A)}$ be a probability distribution on the shares $\mathcal{S}(A)$ given to a subset $A \subset \mathcal{P}$. Usually a secret $K$ is chosen from $\mathcal{K}$ with the uniform distribution. A secret sharing scheme is perfect if

$$
H(K \mid A)= \begin{cases}0 & (\text { if } A \in \Gamma) \\ H(K) & (\text { if } A \notin \Gamma)\end{cases}
$$

where $H(K)$ and $H(K \mid A)$ denote the entropy of $p_{\mathcal{K}}$ and the conditional entropy defined by the joint probability distribution $p_{\mathcal{K} \times \mathcal{S}(A)}$, respectively.

In general, the efficiency of a perfect secret sharing scheme is measured by the information rate $\rho$ [17] defined as

$$
\begin{aligned}
& \rho=\min \left\{\rho_{i}: 1 \leq i \leq n\right\}, \\
& \rho_{i}=\frac{\log |\mathcal{K}|}{\log \left|\mathcal{S}\left(P_{i}\right)\right|}
\end{aligned}
$$

where $\mathcal{S}\left(P_{i}\right)$ denotes the set of possible shares that $P_{i}$ might receive. Obviously, a high information rate is desirable. A perfect secret sharing scheme is ideal if $\rho=1$. Throughout the paper, $p$ is a large prime, and let $Z_{p}$ be a finite field with $p$ elements. In this paper, we assume $\mathcal{K}=\mathcal{S}=Z_{p}$.

\section{B. Shamir's Threshold Scheme}

Shamir's $(k, n)$-threshold scheme is described as follows [1]:

1) A dealer $D$ chooses $n$ distinct nonzero elements of $Z_{p}$, denoted by $x_{1}, x_{2}, \cdots, x_{n}$. The values $x_{i}$ are public.

2) Suppose $D$ wants to share a secret $K \in Z_{p}, D$ chooses $k-1$ elements $a_{1}, a_{2}, \cdots, a_{k-1}$ from $Z_{p}$ independently with the uniform distribution.

3) $D$ distributes the share $s_{i}=f\left(x_{i}\right)$ to $P_{i}(1 \leq i \leq n)$, where

$$
f(x)=K+a_{1} x+a_{2} x^{2}+\cdots+a_{k-1} x^{k-1}
$$

is a polynomial over $Z_{p}$.

It is known that Shamir's $(k, n)$-threshold scheme is perfect and ideal [17], [18]. This implies that every $k$ participants can recover the secret $K$, but no group of less than $k$ participants can get any information about the secret.

The minimal access structure of $(k, n)$-threshold scheme is described as follows:

$$
\Gamma_{0}=\left\{A \in 2^{\mathcal{P}}:|A|=k\right\}
$$

\section{Secret Sharing Scheme Based on Complete Multipartite Graph}

Let $\mathcal{P}=\left\{P_{1}, P_{2}, \cdots, P_{n}\right\}$. Suppose $\mathcal{P}$ can be partitioned into subsets $V_{1}, \cdots, V_{l}$ and $\Gamma_{0}$ can be formed by

$$
\Gamma_{0}=\left\{\{x, y\}: x \in V_{i}, y \in V_{j}, 1 \leq i, j \leq l, i \neq j\right\} .
$$

Then there is an ideal secret sharing scheme realizing the access structure. In this case, we can obtain a complete multipartite graph with vertex set $\mathcal{P}$ and edge set $\Gamma_{0}$. Actually, we can realize the access structure as follows.

1) Compute $l$ shares $s_{1}, s_{2}, \cdots, s_{l}$ by using a $(2, l)$-threshold scheme with $K$ as a secret.

2) $s_{i}$ is assigned to each $P \in V_{i}(1 \leq i \leq l)$.

\section{General Secret Sharing Schemes}

For $\mathcal{P}=\left\{P_{1}, P_{2}, \cdots, P_{n}\right\}, K \in \mathcal{K}$ and $\Gamma$, Benaloh and Leichter's scheme [12] is described as follows.

Benaloh and Leichter's scheme:

1) Let $\Gamma_{0}=\left\{A_{1}, A_{2}, \cdots, A_{m}\right\}$. For $A_{i} \in \Gamma_{0}$, compute $\left|A_{i}\right|$ shares

$$
s_{i, 1}, s_{i, 2}, \cdots, s_{i,\left|A_{i}\right|}
$$

by using an $\left(\left|A_{i}\right|,\left|A_{i}\right|\right)$-threshold scheme with $K$ as a secret independently for $1 \leq i \leq m$.

2) One distinct share from

$$
s_{i, 1}, s_{i, 2}, \cdots, s_{i,\left|A_{i}\right|}
$$

is assigned to each $P \in A_{i}(1 \leq i \leq m)$.

Example 1: For $\mathcal{P}=\left\{P_{1}, P_{2}, P_{3}, P_{4}, P_{5}\right\}$, consider the following access structure

$$
\Gamma_{0}=\left\{A_{1}, A_{2}, \cdots, A_{7}\right\}
$$

where

$$
\begin{aligned}
& A_{1}=\left\{P_{1}, P_{2}\right\}, \\
& A_{2}=\left\{P_{1}, P_{3}\right\}, \\
& A_{3}=\left\{P_{2}, P_{3}\right\}, \\
& A_{4}=\left\{P_{1}, P_{4}\right\}, \\
& A_{5}=\left\{P_{2}, P_{4}\right\}, \\
& A_{6}=\left\{P_{3}, P_{5}\right\}, \\
& A_{7}=\left\{P_{4}, P_{5}\right\} .
\end{aligned}
$$

We shall realize this access structure by Benaloh and Leichter's scheme. In this case, shares are distributed as follows:

$$
\begin{aligned}
& P_{1}: s_{1,1}, s_{2,1}, s_{4,1}, \\
& P_{2}: s_{1,2}, s_{3,1}, s_{5,1}, \\
& P_{3}: s_{2,2}, s_{3,2}, s_{6,1}, \\
& P_{4}: s_{4,2}, s_{5,2}, s_{7,1}, \\
& P_{5}: s_{6,2}, s_{7,2},
\end{aligned}
$$

where $s_{i, j}$ is computed by using Shamir's $\left(\left|A_{i}\right|,\left|A_{i}\right|\right)$-threshold scheme with $K$ as a secret $(1 \leq i \leq$ $\left.7,1 \leq j \leq\left|A_{i}\right|\right)$.

In this example, $14 / 5$ shares are distributed on average. This scheme executes one threshold scheme for each minimal authorized subset. A disadvantage of this scheme is that the number of shares distributed to each participant becomes large as the size of $\Gamma_{0}$ gets large.

For $\mathcal{P}=\left\{P_{1}, P_{2}, \cdots, P_{n}\right\}, K \in \mathcal{K}$ and $\Gamma$, Ito, Saito and Nishizeki's scheme [11] is described as follows.

Ito, Saito and Nishizeki's scheme:

1) Let $\bar{\Gamma}_{1}=\left\{B_{1}, B_{2}, \cdots, B_{t}\right\}$. Compute $t\left(=\left|\bar{\Gamma}_{1}\right|\right)$ shares 


$$
S=\left\{w_{1}, w_{2}, \cdots, w_{t}\right\}
$$

for the secret $K$ by using Shamir's $(t, t)$-threshold scheme.

2) Distribute shares to $P_{i} \in \mathcal{P}(1 \leq i \leq n)$ according to the function $g_{I S N}: \mathcal{P} \rightarrow 2^{S}$ defined as

$$
\begin{aligned}
g_{I S N}\left(P_{i}\right) & =\left\{w_{j}: P_{i} \notin B_{j} \in \bar{\Gamma}_{1}, 1 \leq j \leq t\right\} \\
= & \bigcup_{\substack{1 \leq j \leq t \\
\boldsymbol{P}_{\boldsymbol{i}} \notin \boldsymbol{B}_{\boldsymbol{j}}}}\left\{\boldsymbol{w}_{\boldsymbol{j}}\right\} . \#(1)
\end{aligned}
$$

Example 2: We shall realize the access structure of Example 1 by Ito, Saito and Nishizeki's scheme. In this case, $\bar{\Gamma}_{1}$ is given by

$$
\bar{\Gamma}_{1}=\left\{B_{1}, B_{2}, B_{3}\right\}
$$

where

$$
\begin{aligned}
& B_{1}=\left\{P_{3}, P_{4}\right\}, \\
& B_{2}=\left\{P_{1}, P_{5}\right\}, \\
& B_{3}=\left\{P_{2}, P_{5}\right\} .
\end{aligned}
$$

1) Since $\left|\bar{\Gamma}_{1}\right|=3$, compute 3 shares

$$
w_{1}, w_{2}, w_{3}
$$

by using a (3,3)-threshold scheme for the secret $K$.

2) According to the function $g_{I S N}$, distribute shares as follows:

$$
\begin{aligned}
& g_{I S N}\left(P_{1}\right)=\left\{w_{1}, w_{3}\right\}, \\
& g_{I S N}\left(P_{2}\right)=\left\{w_{1}, w_{2}\right\}, \\
& g_{I S N}\left(P_{3}\right)=\left\{w_{2}, w_{3}\right\}, \\
& g_{I S N}\left(P_{4}\right)=\left\{w_{2}, w_{3}\right\}, \\
& g_{I S N}\left(P_{5}\right)=\left\{w_{1}\right\} .
\end{aligned}
$$

In this scheme, to recover the secret a group $X \subset \mathcal{P}$ need to collect all shares. If $\mathrm{X} \subset B_{j} \in \bar{\Gamma}_{1}, X$ cannot collect the share $w_{j}$. On the other hand, If $X \in \Gamma$, then there exists $P \in X$ such that $P \in X-B_{j}$ for all $B_{j}(1 \leq j \leq t)$. Thus, $X$ can collect $w_{1}, \cdots, w_{t}$ and recover the secret. In this example, $9 / 5$ shares are distributed on average. This scheme needs one share for each maximal unauthorized subset. Thus this scheme needs $\left|\bar{\Gamma}_{1}\right|$ shares in total. A disadvantage of this scheme is that the number of shares distributed to each participant becomes large as the size of $\bar{\Gamma}_{1}$ gets large.

\section{Proposed SCHEME}

Here, we modify Benaloh and Leichter's scheme [12] and propose a new secret sharing scheme realizing general access structures, which is based on authorized subsets. The proposed scheme is perfect and can reduce the number of shares distributed to one specified participant $P^{\prime} \in \mathcal{P}$ by dividing into $\Gamma_{0}$ according to $P^{\prime}$. Furthermore, we can apply the proposed scheme to the same access structure recursively. The proposed scheme is more efficient than or equal to Benaloh and Leichter's scheme [12] for any access structure.

For $\mathcal{P}=\left\{P_{1}, P_{2}, \cdots, P_{n}\right\}, P^{\prime} \in \mathcal{P}, K \in \mathcal{K}$ and $\Gamma$, the proposed scheme is described as follows.

Proposed Scheme:

1) Let $\Gamma_{0}^{(1)}=\left\{A \subset \mathcal{P}-\left\{P^{\prime}\right\}: A \cup\left\{P^{\prime}\right\} \in \Gamma_{0}\right\} \quad$ and represent it as

$$
\Gamma_{0}^{(1)}=\left\{A_{1}^{(1)}, A_{2}^{(1)}, \cdots, A_{l}^{(1)}\right\}
$$

2) Let $\Gamma_{0}^{(0)}=\left\{A \in \Gamma_{0}: P^{\prime} \notin A\right\}$ and represent it as

$$
\Gamma_{0}^{(0)}=\left\{A_{1}^{(0)}, A_{2}^{(0)}, \cdots, A_{m}^{(0)}\right\}
$$

3) compute 2 shares

$$
S=\left\{w_{1}, w_{2}\right\}
$$

by using Shamir's $(2,2)$-threshold scheme with $K$ as a secret $w_{2}$ is assigned to $P^{\prime} \in \mathcal{P}$.

4) For $A_{i}^{(1)} \in \Gamma_{0}^{(1)}$, compute $\left|A_{i}^{(1)}\right|$ shares

$$
S_{1, i}=\left\{s_{1, i, 1}, s_{1, i, 2}, \cdots, s_{1, i,\left|A_{i}^{(1)}\right|}\right\}
$$

by using Shamir's $\left(\left|A_{i}^{(1)}\right|,\left|A_{i}^{(1)}\right|\right)$-threshold scheme with $w_{1}$ as a secret independently for $1 \leq i \leq l$. One distinct share from $S_{1, i}$ is assigned to each

$$
P \in A_{i}^{(1)}(1 \leq i \leq l)
$$

5) For $A_{i}^{(0)} \in \Gamma_{0}^{(0)}$, compute $\left|A_{i}^{(0)}\right|$ shares

$$
S_{0, i}=\left\{s_{0, i, 1}, s_{0, i, 2}, \cdots, s_{0, i,\left|A_{i}^{(0)}\right|}\right\}
$$

by using Shamir's $\left(\left|A_{i}^{(0)}\right|,\left|A_{i}^{(0)}\right|\right)$-threshold scheme with $K$ as a secret independently for $1 \leq i \leq m$. One distinct share from $S_{0, i}$ is assigned to each

$$
P \in A_{i}^{(0)}(1 \leq i \leq m)
$$

Example 3: Let $P^{\prime}=P_{1}$ and we shall realize the access structure of Example 1 by the proposed scheme.

1) $\Gamma_{0}^{(1)}$ is defined by

$$
\Gamma_{0}^{(1)}=\left\{A_{1}^{(1)}, A_{2}^{(1)}, A_{3}^{(1)}\right\}
$$

where

$$
\begin{aligned}
& A_{1}^{(1)}=\left\{P_{2}\right\} \\
& A_{2}^{(1)}=\left\{P_{3}\right\} \\
& A_{3}^{(1)}=\left\{P_{4}\right\}
\end{aligned}
$$

2) $\Gamma_{0}^{(0)}$ is defined by

$$
\Gamma_{0}^{(0)}=\left\{A_{1}^{(0)}, A_{2}^{(0)}, A_{3}^{(0)}, A_{4}^{(0)}\right\}
$$

where

$$
\begin{aligned}
& A_{1}^{(0)}=\left\{P_{2}, P_{3}\right\}, \\
& A_{2}^{(0)}=\left\{P_{2}, P_{4}\right\},
\end{aligned}
$$




$$
\begin{aligned}
& A_{3}^{(0)}=\left\{P_{3}, P_{5}\right\}, \\
& A_{4}^{(0)}=\left\{P_{4}, P_{5}\right\} .
\end{aligned}
$$

3) compute 2 shares $w_{1}, w_{2}$ by using Shamir's $(2,2)$-threshold scheme with $K$ as a secret. $w_{2}$ is assigned to $P_{1}$.

4) In this case, $\left|A_{1}^{(1)}\right|=\left|A_{2}^{(1)}\right|=\left|A_{3}^{(1)}\right|=1$. For $A_{i}^{(1)} \in \Gamma_{0}^{(1)}$, set

$$
s_{1, i, 1}=w_{1}
$$

and $s_{1, i, 1}$ is assigned to $P \in A_{i}^{(1)}(1 \leq i \leq 3)$.

5) In this case, $\left|A_{1}^{(0)}\right|=\left|A_{2}^{(0)}\right|=\left|A_{3}^{(0)}\right|=\left|A_{4}^{(0)}\right|=2$. For $A_{i}^{(0)} \in \Gamma_{0}^{(0)}$, compute 2 shares

$$
S_{0, i}=\left\{s_{0, i, 1}, S_{0, i, 2}\right\}
$$

by using Shamir's $(2,2)$-threshold scheme with $K$ as a secret independently for $1 \leq i \leq 4$. One distinct share from $S_{0, i}$ is assigned to each $P \in A_{i}^{(0)}(1 \leq i \leq 4)$.

6) In this case, shares are distributed as follows:

$$
\begin{aligned}
& P_{1}: w_{2}, \\
& P_{2}: s_{1,1,1}, s_{0,1,1}, s_{0,2,1}, \\
& P_{3}: s_{1,2,1}, s_{0,1,2}, s_{0,3,1}, \\
& P_{4}: s_{1,3,1}, s_{0,2,2}, s_{0,4,1}, \\
& P_{5}: s_{0,3,2}, s_{0,4,2} .
\end{aligned}
$$

In this example, the proposed scheme can reduce the number of shares distributed to $P_{1} \in \mathcal{P}$. Actually, the proposed scheme distributes $12 / 5$ shares on average, which is smaller than 14/5 achieved by Benaloh and Leichter's scheme. Hence, the proposed scheme is more efficient than Benaloh and Leichter's scheme.

The next theorem shows that the proposed scheme is perfect.

Theorem 1: For $\mathcal{P}=\left\{P_{1}, P_{2}, \cdots, P_{n}\right\}, P^{\prime} \in \mathcal{P}$ and any access structure $\Gamma\left(\subset 2^{\mathcal{P}}\right)$, distribute shares for a secret $K$ by using the proposed scheme. Then, for any subset $\mathrm{X} \subset \mathcal{P}$,

(a) $X \in \Gamma \Rightarrow H(K \mid X)=0$,

(b) $X \notin \Gamma \Rightarrow H(K \mid X)=H(K)$.

Proof: Let $X_{S}, X_{S_{1, i}}$ and $X_{S_{0, j}}$ denote the shares in $S-$ $\left\{w_{1}\right\}, S_{1, i}$ and $S_{0, j}$ assigned to $X$, respectively $(1 \leq i \leq l, 1 \leq$ $j \leq m)$. At first, we show $H(K \mid X)=0$ for any $X \in \Gamma$.

(Case i) $P^{\prime} \notin X$ : From the property of access structure and the definition of $\Gamma_{0}^{(0)}$, there exists $A_{i}^{(0)} \in \Gamma_{0}^{(0)}$ such that $A_{i}^{(0)} \subset X$. In this case, we have

$$
\left|X_{S_{0, i}}\right|=\left|S_{0, i}\right|
$$

Since $s_{0, i, 1}, s_{0, i, 2}, \cdots, s_{0, i, \mid A_{i}^{(0)}}$ are shares computed by Shamir's $\left(\left|A_{i}^{(0)}\right|,\left|A_{i}^{(0)}\right|\right)$-threshold scheme with $K$ as a secret, we have

$$
\begin{aligned}
& H(K \mid X) \\
& \quad=H\left(K \mid X_{S}, X_{S_{1,1}}, \cdots, X_{S_{1, l}}, X_{S_{0,1}}, \cdots, X_{S_{0, m}}\right)
\end{aligned}
$$

$$
\begin{aligned}
\leq & H\left(K \mid X_{S_{0, i}}\right) \\
& =\mathbf{0} .
\end{aligned}
$$

(Case ii) $P^{\prime} \in X$ : From the property of access structure and the definition of $\Gamma_{0}^{(1)}$, there exists $A_{i}^{(1)} \in \Gamma_{0}^{(1)}$ such that $A_{i}^{(1)} \subset X$. In this case, we have

$$
\left|X_{S}\right|=1 \text { and }\left|X_{S_{1, i}}\right|=\left|S_{1, i}\right| \text {. }
$$

Since $s_{1, i, 1}, s_{1, i, 2}, \cdots, s_{1, i,\left|A_{i}^{(1)}\right|}$ are shares computed by Shamir's $\left(\left|A_{i}^{(1)}\right|,\left|A_{i}^{(1)}\right|\right)$-threshold scheme with $w_{1}$ as a secret, we have

$$
\begin{aligned}
& H(K \mid X) \\
& \quad=H\left(K \mid X_{S}, X_{S_{1,1}}, \cdots, X_{S_{1, l}}, X_{S_{0,1}}, \cdots, X_{S_{0, m}}\right) \\
& \quad \leq H\left(K \mid X_{S}, X_{S_{0, i}}\right) \\
& \quad=\mathbf{0} .
\end{aligned}
$$

Since $H(K \mid X) \geq 0$ is obvious, we have

$$
H(K \mid X)=0
$$

for any $X \in \Gamma$ from (2) and (3).

Next we show $H(K \mid X)=H(K)$ for any $X \notin \Gamma$.

(Case i) $P^{\prime} \notin X$ : From the property of the access structure and the definition of $\Gamma_{0}^{(0)}$ and $\Gamma_{0}^{(1)}$, we have

$$
A_{i}^{(0)} \not \subset X(1 \leq i \leq m)
$$

and

$$
\left|X_{S}\right|=\mathbf{0}
$$

(Case ii) $P^{\prime} \in X$ : From the property of access structure and the definition of $\Gamma_{0}^{(0)}$ and $\Gamma_{0}^{(1)}$, we have

$$
A_{i}^{(0)} \not \subset X(1 \leq i \leq m)
$$

and

$$
A_{i}^{(1)} \not \subset X(1 \leq i \leq l)
$$

From (4) and (6), we have

$$
H\left(K \mid X_{S_{0, i}}\right)=H(K)
$$

for any $X \notin \Gamma(1 \leq i \leq m)$. This implies

$$
H\left(X_{S_{0, i}} \mid K\right)=H\left(X_{S_{0, i}}\right)
$$

for any $X \notin \Gamma(1 \leq i \leq m)$. From (5) and (7), we have

$$
\boldsymbol{H}\left(\boldsymbol{K} \mid \boldsymbol{X}_{S}, \boldsymbol{X}_{S_{1, i}}\right)=\boldsymbol{H}(\boldsymbol{K})
$$

for any $X \notin \Gamma(1 \leq i \leq l)$. From the definition of $S$, $S_{1,1}, \cdots, S_{1, l}$ and (9), we have 


$$
H\left(K \mid X_{S}, X_{S_{1,1}}, X_{S_{1,2}}, \cdots, X_{S_{1, l}}\right)=H(K) .
$$

This implies

$$
\begin{aligned}
& H\left(X_{S}, X_{S_{1,1}}, X_{S_{1,2}}, \cdots, X_{S_{1, l}} \mid K\right) \\
& \quad=\boldsymbol{H}\left(\boldsymbol{X}_{\boldsymbol{S}}, \boldsymbol{X}_{\boldsymbol{S}_{\mathbf{1 , 1}}}, \boldsymbol{X}_{\boldsymbol{S}_{\mathbf{1}, \mathbf{2}}}, \cdots, \boldsymbol{X}_{\boldsymbol{S}_{\mathbf{1}, l}}\right) .
\end{aligned}
$$

In order to show $H(K \mid X)=H(K)$, we expand $H(K \mid X)$ as follows:

$$
\begin{aligned}
& H(K \mid X) \\
& =H\left(K \mid X_{S}, X_{S_{1,1}}, \cdots, X_{S_{1, l}}, X_{S_{0,1}}, \cdots, X_{S_{0, m}}\right) \\
& =H(K) \\
& \quad+H\left(X_{S}, X_{S_{1,1}}, \cdots, X_{S_{1, l}}, X_{S_{0,1}}, \cdots, X_{S_{0, m}} \mid K\right) \\
& \quad-\quad H\left(\boldsymbol{X}_{\boldsymbol{S}}, \boldsymbol{X}_{\boldsymbol{S}_{1, \mathbf{1}}}, \cdots, \boldsymbol{X}_{\boldsymbol{S}_{1, l}} \boldsymbol{X}_{\boldsymbol{S}_{\mathbf{0}, \mathbf{1}}}, \cdots, \boldsymbol{X}_{\boldsymbol{S}_{\mathbf{0}, \boldsymbol{m}}}\right) .
\end{aligned}
$$

From the chain rule for entropy, we have

$$
\begin{aligned}
& H\left(X_{S}, X_{S_{1,1}}, \cdots, X_{S_{1, l}}, X_{S_{0,1}}, \cdots, X_{S_{0, m}} \mid K\right) \\
& =H\left(X_{S}, X_{S_{1,1}}, \cdots, X_{S_{1, l}} \mid K\right) \\
& +\sum_{t=1}^{m} H\left(X_{S_{0, t}} \mid K, X_{S}, X_{S_{1,1}}, \cdots\right. \\
& \text { (*) } \left.\quad \cdots, X_{S_{1, l}}, X_{S_{0,1}}, \cdots, X_{S_{0, t-1}}\right) \\
& \stackrel{(*)}{=} H\left(X_{S}, X_{S_{1,1}} \cdots, X_{S_{1, l}} \mid K\right) \\
& +\sum_{t=1}^{m} H\left(X_{S_{0, t}} \mid K\right) \\
& =H\left(X_{S}, X_{S_{1,1}}, \cdots, X_{S_{1, l}}\right) \\
& +\sum_{t=1}^{m} \boldsymbol{H}\left(\boldsymbol{X}_{S_{0, t}}\right) \text {. }
\end{aligned}
$$

Here, $(*)$ comes from the definition of $\Gamma_{0}^{(0)}$ and $\Gamma_{0}^{(1)}$ and the fact that $X_{S_{0,1}}, \cdots, X_{S_{0, m}}$ are mutually independent and the last equality comes from (8) and (10). On the other hand, we have

$$
\begin{gathered}
H\left(X_{S}, X_{S_{1,1}}, \cdots, X_{S_{1, l}}, X_{S_{0,1}}, \cdots, X_{S_{0, m}}\right) \\
=H\left(X_{S}, X_{S_{1,1}} \cdots, X_{S_{1, l}}\right) \\
\quad+\sum_{t=1}^{m} H\left(X_{S_{0, t}} X_{S}, X_{S_{1,1}}, \cdots\right. \\
\left.\cdots, X_{S_{1, l}}, X_{S_{0,1}}, \cdots, X_{S_{0, t-1}}\right) \\
\leq \quad H\left(X_{S}, X_{S_{1,1}} \cdots, X_{S_{1, l}}\right) \\
\quad+\sum_{t=\mathbf{1}}^{m} \boldsymbol{H}\left(\boldsymbol{X}_{\boldsymbol{S}_{\mathbf{0}, t}}\right) .
\end{gathered}
$$

Substituting (12) and (13) into (11), we obtain $H(K \mid X) \geq$ $H(K)$. Since $H(K \mid X) \leq H(K)$ is obvious, we have

$$
H(K \mid X)=H(K) .
$$

Let $N(P)$ be the number of shares distributed to $P \in \mathcal{P}$ by using the proposed scheme. Similarly, let $N_{B L}(P)$ be the number of shares distributed to $P \in \mathcal{P}$ by using Benaloh and Leichter's scheme. The next theorem shows the proposed scheme is more efficient than Benaloh and Leichter's scheme from the viewpoint of the number of shares distributed to $P^{\prime} \in \mathcal{P}$.
Theorem 2: For any $P \in \mathcal{P}$, the number of shares distributed to $P$ is evaluated as follows:

$$
N(P)= \begin{cases}N_{B L}(P)-l+1 & \left(P=P^{\prime}\right) \\ N_{B L}(P) & \left(P \neq P^{\prime}\right) .\end{cases}
$$

Proof: From the definition of $\Gamma_{0}^{(1)}$, we have

$$
\left(P^{\prime}\right)=\mathbf{1}
$$

and

$$
\left\{X \in \Gamma_{0}: P^{\prime} \in X\right\}=\left\{X \cup\left\{P^{\prime}\right\}: X \in \Gamma_{0}^{(1)}\right\} .
$$

$N_{B L}(P)$ is obtain by

$$
\begin{aligned}
N_{B L}\left(P^{\prime}\right)= & \left|\left\{X \in \Gamma_{0}: P^{\prime} \in X\right\}\right| \\
= & \left|\left\{X \cup\left\{P^{\prime}\right\}: X \in \Gamma_{0}^{(1)}\right\}\right| \\
= & \left|\Gamma_{0}^{(1)}\right| \\
& =\boldsymbol{l} .
\end{aligned}
$$

On the other hand, for $P \neq P^{\prime}$, we have

$$
\boldsymbol{N}(\boldsymbol{P})=\left|\left\{X \in \Gamma_{0}^{(0)}: P \in X\right\}\right|+\left|\left\{X \in \Gamma_{0}^{(1)}: P \in X\right\}\right| .
$$

and

$$
\begin{aligned}
N_{B L}(P)= & \left|\left\{X \in \Gamma_{0}: P \in X\right\}\right| \\
= & \left|\left\{X \in \Gamma_{0}^{(0)}: P \in X\right\}\right| \\
& +\left|\left\{X \cup\left\{P^{\prime}\right\}: P \in X \in \Gamma_{0}^{(1)}\right\}\right| \\
= & \left|\left\{X \in \Gamma_{0}^{(0)}: P \in X\right\}\right| \\
& +\left|\left\{\boldsymbol{X} \in \Gamma_{\mathbf{0}}^{(\mathbf{1})}: \boldsymbol{P} \in \boldsymbol{X}\right\}\right| .
\end{aligned}
$$

Theorem 2 is easily obtained by (14)-(17).

We can apply the proposed scheme to the same access structure recursively. That is, the proposed scheme can reduce the number of shares distributed to another participant once again by applying the proposed scheme recursively.

Example 4: We shall apply the proposed scheme to the access structure of Example 1 recursively.

1) Let $P^{\prime \prime}=P_{2}$ and apply the proposed scheme to $\Gamma_{0}^{(0)}$ of Example 3 again. In this case, $\Gamma_{0}^{(01)}$ is defined by

$$
\Gamma_{0}^{(01)}=\left\{A_{1}^{(01)}, A_{2}^{(01)}\right\}
$$

where

$$
\begin{aligned}
& A_{1}^{(01)}=\left\{P_{3}\right\}, \\
& A_{2}^{(01)}=\left\{P_{4}\right\} .
\end{aligned}
$$

2) $\Gamma_{0}^{(00)}$ is defined by

$$
\Gamma_{0}^{(00)}=\left\{A_{1}^{(00)}, A_{2}^{(00)}\right\}
$$

where

$$
A_{1}^{(00)}=\left\{P_{3}, P_{5}\right\},
$$




$$
A_{2}^{(00)}=\left\{P_{4}, P_{5}\right\}
$$

3) compute 2 shares $w_{1}^{\prime}, w_{2}^{\prime}$ by using Shamir's $(2,2)$-threshold scheme with $K$ as a secret. $w_{2}^{\prime}$ is assigned to $P_{2}$.

4) Since $\left|A_{1}^{(01)}\right|=\left|A_{2}^{(01)}\right|=1$, set

$$
s_{1, i, 1}^{\prime}=w_{1}^{\prime}
$$

and $s_{1, i, 1}^{\prime}$ is assigned to $P \in A_{i}^{(01)}(1 \leq i \leq 2)$.

5) Let $P^{\prime \prime \prime}=P_{5}$ and apply the proposed scheme to $\Gamma_{0}^{(00)}$ again. In this case, $\Gamma_{0}^{(001)}$ is defined by

$$
\Gamma_{0}^{(001)}=\left\{A_{1}^{(001)}, A_{2}^{(001)}\right\}
$$

where

$$
\begin{aligned}
& A_{1}^{(001)}=\left\{P_{3}\right\}, \\
& A_{2}^{(001)}=\left\{P_{4}\right\}
\end{aligned}
$$

and $\Gamma_{0}^{(000)}=\phi$.

6) compute 2 shares $w_{1}^{\prime \prime}, w_{2}^{\prime \prime}$ by using Shamir's $(2,2)$-threshold scheme with $K$ as a secret. $w_{2}^{\prime \prime}$ is assigned to $P_{5}$.

7) $\quad$ Since $\left|A_{1}^{(001)}\right|=\left|A_{2}^{(001)}\right|=1$, set

$$
s_{1, i, 1}^{\prime \prime}=w_{1}^{\prime \prime}
$$

and $s_{1, i, 1}^{\prime \prime}$ is assigned to $P \in A_{i}^{(001)}(1 \leq i \leq 2)$.

8) In this case, shares are distributed as follows:

$$
\begin{aligned}
& P_{1}: w_{2}, \\
& P_{2}: s_{1,1,1}, w_{2}^{\prime}, \\
& P_{3}: s_{1,2,1}, s_{1,1,1}^{\prime}, s_{1,1,1}^{\prime \prime}, \\
& P_{4}: s_{1,3,1}, s_{1,2,1}^{\prime}, s_{1,2,1}^{\prime \prime}, \\
& P_{5}: w_{2}^{\prime \prime} .
\end{aligned}
$$

In this example, the proposed scheme can reduce the number of shares distributed to $P_{2}, P_{5}$ as well as $P_{1}$. Actually, the proposed scheme distributes 2 shares on average.

The proposed scheme can yet reduce the number of shares distributed to each participant if $\Gamma_{1}^{(0)}$ or $\Gamma_{0}^{(0)}$ forms a complete multipartite graph. by

Example 5: From Example 4, $\Gamma_{0}^{(0)}$ of Example 3 is denoted

$$
\Gamma_{0}^{(0)}=V_{1} \cup V_{2}
$$

where

$$
\begin{aligned}
& V_{1}=\left\{P_{2}, P_{5}\right\}, \\
& V_{2}=\left\{P_{3}, P_{4}\right\} .
\end{aligned}
$$

In this case, shares are distributed as follows:

$$
\begin{aligned}
& P_{1}: w_{2}, \\
& P_{2}: s_{1,1,1}, w_{2}^{\prime}, \\
& P_{3}: s_{1,2,1}, s_{1,1,1}^{\prime}, \\
& P_{4}: s_{1,3,1}, s_{1,2,1}^{\prime}, \\
& P_{5}: w_{2}^{\prime} .
\end{aligned}
$$

In this example, the proposed scheme can reduce the number of shares distributed to $P_{3}, P_{4}$ besides $P_{1}, P_{2}, P_{5}$. Actually, the proposed scheme distributes $8 / 5$ shares on average.

\section{EVALUATION OF EFFICIENCY}

Here, we consider the efficiency of the proposed scheme. Let $N^{\prime}(P)$ be the number of shares distributed to $P \in \mathcal{P}$ by using the proposed scheme recursively. Similarly, let $N^{\prime \prime}(P)$ be the number of shares distributed to $P \in \mathcal{P}$ by using the property of complete multipartite graphs and the proposed scheme recursively. Here, we denote the total number of shares distributed to all participants by

$$
\begin{aligned}
N_{B L} & =\sum_{P \in \mathcal{P}} N_{B L}(P), \\
N & =\sum_{P \in \mathcal{P}} N(P), \\
N^{\prime} & =\sum_{P \in \mathcal{P}} N^{\prime}(P), \\
N^{\prime \prime} & =\sum_{P \in \mathcal{P}} N^{\prime \prime}(P), \\
g_{I S N} & =\sum_{P \in \mathcal{P}} g_{I S N}(P) .
\end{aligned}
$$

From Theorem 3 and the definition of $\Gamma_{0}^{(0)}$ and $\Gamma_{0}^{(1)}$, we have

$$
N^{\prime \prime} \leq N^{\prime} \leq N_{B L} .
$$

For all 180 access structures on five participants clarified by Jackson and Martin [19], we obtain $N_{B L}, N, N^{\prime}, N^{\prime \prime}$ and $g_{I S N}$ in order to evaluate the efficiency of the proposed scheme. Table I shows the number of shares distributed to participants by these five schemes. We summarize the comparison among $N_{B L}, N, N^{\prime}$ and $N^{\prime \prime}$ in Table II. For 173 access structures, $N$ is smaller than $N_{B L}$. For 143 access structures, we can reduce the number of shares distributed to participants by applying the proposed scheme recursively. The proposed scheme can yet reduce the number of shares distributed to each participant by using the property of complete multipartite graphs for 40 access structures.

We summarize the comparison among between $N^{\prime \prime}$ and $g_{I S N}$ in Table III. For 169 access structures, $N^{\prime \prime}$ is smaller than $g_{I S N}$. There is no access structure for which Ito, Saito and Nishizeki's scheme is better. 
TABLE I: COMPARISON OF THE NUMBER OF SHARES DISTRIBUTED TO PARTICIPANTS

\begin{tabular}{|c|c|c|c|c|c|}
\hline \# & $N_{B L}$ & $N$ & $N^{\prime}$ & $N^{\prime \prime}$ & $g_{I S N}$ \\
\hline 1 & 5 & 5 & 5 & 5 & 5 \\
\hline 2 & 5 & 5 & 5 & 5 & 8 \\
\hline 3 & 6 & 5 & 5 & 5 & 7 \\
\hline 4 & 8 & 7 & 7 & 7 & 12 \\
\hline 5 & 5 & 5 & 5 & 5 & 9 \\
\hline 6 & 9 & 7 & 7 & 7 & 10 \\
\hline 7 & 11 & 9 & 8 & 7 & 11 \\
\hline 8 & 13 & 11 & 10 & 10 & 16 \\
\hline 9 & 7 & 5 & 5 & 5 & 6 \\
\hline 10 & 10 & 8 & 8 & 8 & 13 \\
\hline 11 & 7 & 6 & 6 & 6 & 9 \\
\hline 12 & 9 & 8 & 7 & 5 & 6 \\
\hline 13 & 8 & 7 & 7 & 7 & 12 \\
\hline 14 & 5 & 5 & 5 & 5 & 12 \\
\hline 15 & 6 & 5 & 5 & 5 & 8 \\
\hline 16 & 9 & 8 & 7 & 7 & 15 \\
\hline 17 & 7 & 6 & 5 & 5 & 7 \\
\hline 18 & 10 & 8 & 7 & 7 & 11 \\
\hline 19 & 13 & 11 & 10 & 10 & 18 \\
\hline 20 & 5 & 5 & 5 & 5 & 8 \\
\hline 21 & 14 & 11 & 10 & 10 & 13 \\
\hline 22 & 16 & 13 & 11 & 10 & 14 \\
\hline 23 & 18 & 15 & 12 & 11 & 15 \\
\hline 24 & 20 & 17 & 14 & 14 & 20 \\
\hline 25 & 12 & 9 & 8 & 8 & 9 \\
\hline 26 & 14 & 11 & 9 & 9 & 10 \\
\hline 27 & 17 & 14 & 12 & 11 & 17 \\
\hline 28 & 14 & 11 & 10 & 9 & 13 \\
\hline 29 & 16 & 13 & 11 & 9 & 10 \\
\hline 30 & 15 & 12 & 11 & 10 & 16 \\
\hline 31 & 12 & 10 & 9 & 8 & 12 \\
\hline 32 & 14 & 12 & 10 & 8 & 9 \\
\hline 33 & 13 & 11 & 10 & 9 & 15 \\
\hline 34 & 10 & 7 & 7 & 7 & 8 \\
\hline 35 & 12 & 9 & 9 & 9 & 16 \\
\hline 36 & 13 & 10 & 9 & 9 & 12 \\
\hline 37 & 16 & 13 & 11 & 11 & 19 \\
\hline 38 & 10 & 8 & 7 & 7 & 8 \\
\hline 39 & 12 & 10 & 9 & 7 & 12 \\
\hline 40 & 13 & 11 & 10 & 10 & 15 \\
\hline 41 & 10 & 8 & 8 & 8 & 15 \\
\hline 42 & 11 & 9 & 8 & 8 & 11 \\
\hline 43 & 14 & 12 & 10 & 10 & 18 \\
\hline 44 & 8 & 7 & 7 & 7 & 18 \\
\hline 45 & 9 & 7 & 7 & 7 & 10 \\
\hline 46 & 12 & 10 & 9 & 9 & 14 \\
\hline 47 & 15 & 13 & 10 & 10 & 21 \\
\hline 48 & 11 & 8 & 8 & 8 & 10 \\
\hline 49 & 14 & 11 & 9 & 9 & 11 \\
\hline 50 & 17 & 14 & 11 & 11 & 15 \\
\hline 51 & 20 & 17 & 14 & 14 & 22 \\
\hline 52 & 11 & 9 & 8 & 8 & 11 \\
\hline 53 & 13 & 11 & 10 & 8 & 15 \\
\hline 54 & 15 & 13 & 11 & 9 & 12 \\
\hline 55 & 14 & 12 & 10 & 10 & 18 \\
\hline 56 & 12 & 10 & 8 & 8 & 10 \\
\hline 57 & 15 & 12 & 10 & 10 & 14 \\
\hline 58 & 18 & 15 & 13 & 13 & 21 \\
\hline 59 & 8 & 5 & 5 & 5 & 5 \\
\hline 60 & 12 & 9 & 9 & 9 & 12 \\
\hline
\end{tabular}

\begin{tabular}{c|r|r|r|r|r}
\hline$\#$ & $N_{B L}$ & \multicolumn{1}{|c|}{$N$} & $N^{\prime}$ & \multicolumn{1}{|c|}{$N^{\prime \prime}$} & $g_{I S N}$ \\
\hline 61 & 8 & 6 & 6 & 6 & 7 \\
\hline 62 & 10 & 8 & 7 & 6 & 8 \\
\hline
\end{tabular}

\begin{tabular}{|c|c|c|c|c|c|}
\hline 126 & 8 & 7 & 7 & 7 & 10 \\
\hline 127 & 11 & 9 & 8 & 8 & 8 \\
\hline 128 & 8 & 7 & 6 & 6 & 10 \\
\hline 129 & 9 & 8 & 7 & 7 & 12 \\
\hline 130 & 5 & 5 & 5 & 5 & 12 \\
\hline 131 & 6 & 5 & 5 & 5 & 7 \\
\hline 132 & 10 & 9 & 7 & 7 & 14 \\
\hline 133 & 15 & 12 & 10 & 10 & 13 \\
\hline 134 & 18 & 14 & 11 & 10 & 14 \\
\hline 135 & 21 & 17 & 12 & 11 & 15 \\
\hline 136 & 24 & 19 & 14 & 13 & 19 \\
\hline 137 & 27 & 22 & 16 & 16 & 23 \\
\hline 138 & 30 & 25 & 19 & 19 & 30 \\
\hline 139 & 21 & 16 & 13 & 13 & 18 \\
\hline 140 & 21 & 17 & 12 & 11 & 15 \\
\hline 141 & 24 & 20 & 14 & 13 & 16 \\
\hline 142 & 18 & 15 & 11 & 11 & 14 \\
\hline 143 & 12 & 9 & 7 & 7 & 8 \\
\hline 144 & 15 & 11 & 8 & 7 & 9 \\
\hline 145 & 18 & 13 & 10 & 10 & 13 \\
\hline 146 & 22 & 17 & 14 & 14 & 20 \\
\hline 147 & 18 & 14 & 11 & 10 & 14 \\
\hline 148 & 21 & 17 & 12 & 11 & 12 \\
\hline 149 & 19 & 15 & 12 & 11 & 16 \\
\hline 150 & 15 & 12 & 10 & 10 & 13 \\
\hline 151 & 18 & 15 & 11 & 9 & 11 \\
\hline 152 & 15 & 12 & 9 & 9 & 13 \\
\hline 153 & 16 & 13 & 11 & 11 & 15 \\
\hline 154 & 12 & 10 & 8 & 8 & 12 \\
\hline 155 & 15 & 12 & 10 & 8 & 10 \\
\hline 156 & 18 & 15 & 11 & 7 & 8 \\
\hline 157 & 13 & 11 & 10 & 10 & 14 \\
\hline 158 & 9 & 7 & 5 & 5 & 5 \\
\hline 159 & 12 & 10 & 8 & 8 & 15 \\
\hline 160 & 13 & 10 & 8 & 8 & 10 \\
\hline 161 & 17 & 14 & 11 & 11 & 17 \\
\hline 162 & 9 & 7 & 6 & 6 & 7 \\
\hline 163 & 12 & 9 & 7 & 5 & 5 \\
\hline 164 & 16 & 13 & 11 & 9 & 12 \\
\hline 165 & 12 & 10 & 8 & 8 & 12 \\
\hline 166 & 15 & 13 & 10 & 10 & 10 \\
\hline 167 & 13 & 11 & 9 & 9 & 14 \\
\hline 168 & 9 & 8 & 7 & 7 & 14 \\
\hline 169 & 10 & 8 & 7 & 7 & 9 \\
\hline 170 & 14 & 12 & 9 & 9 & 16 \\
\hline 171 & 6 & 5 & 5 & 5 & 9 \\
\hline 172 & 10 & 9 & 8 & 8 & 16 \\
\hline 173 & 7 & 6 & 5 & 5 & 6 \\
\hline 174 & 11 & 9 & 7 & 7 & 11 \\
\hline 175 & 15 & 13 & 10 & 10 & 18 \\
\hline 176 & 8 & 7 & 5 & 5 & 5 \\
\hline 177 & 12 & 10 & 7 & 7 & 8 \\
\hline 178 & 16 & 13 & 10 & 10 & 13 \\
\hline 179 & 20 & 17 & 14 & 14 & 20 \\
\hline 180 & 5 & 5 & 5 & 5 & 5 \\
\hline
\end{tabular}

\begin{tabular}{|c|c|c|c|c|c|}
\hline 63 & 12 & 10 & 8 & 5 & 5 \\
\hline 64 & 11 & 9 & 8 & 8 & 11 \\
\hline 65 & 9 & 7 & 7 & 7 & 9 \\
\hline 66 & 12 & 10 & 8 & 8 & 10 \\
\hline 67 & 15 & 13 & 10 & 10 & 14 \\
\hline 68 & 10 & 8 & 8 & 8 & 11 \\
\hline 69 & 11 & 9 & 8 & 7 & 11 \\
\hline 70 & 14 & 12 & 11 & 9 & 18 \\
\hline 71 & 8 & 7 & 6 & 6 & 11 \\
\hline 72 & 10 & 9 & 8 & 8 & 15 \\
\hline 73 & 11 & 9 & 9 & 9 & 18 \\
\hline 74 & 9 & 7 & 7 & 7 & 10 \\
\hline 75 & 12 & 10 & 9 & 9 & 14 \\
\hline 76 & 15 & 13 & 11 & 11 & 21 \\
\hline 77 & 12 & 10 & 8 & 8 & 14 \\
\hline 78 & 9 & 8 & 7 & 7 & 7 \\
\hline 79 & 10 & 8 & 7 & 7 & 9 \\
\hline 80 & 12 & 10 & 9 & 9 & 21 \\
\hline 81 & 13 & 11 & 9 & 9 & 13 \\
\hline 82 & 16 & 13 & 11 & 11 & 17 \\
\hline 83 & 19 & 16 & 13 & 13 & 24 \\
\hline 84 & 13 & 10 & 9 & 9 & 13 \\
\hline 85 & 16 & 13 & 11 & 11 & 17 \\
\hline 86 & 9 & 8 & 7 & 7 & 14 \\
\hline 87 & 10 & 8 & 8 & 8 & 12 \\
\hline 88 & 13 & 11 & 9 & 9 & 13 \\
\hline 89 & 16 & 13 & 10 & 10 & 17 \\
\hline 90 & 10 & 8 & 7 & 7 & 9 \\
\hline 91 & 13 & 11 & 9 & 9 & 10 \\
\hline 92 & 6 & 5 & 5 & 5 & 10 \\
\hline 93 & 7 & 5 & 5 & 5 & 7 \\
\hline 94 & 10 & 8 & 7 & 7 & 12 \\
\hline 95 & 13 & 11 & 8 & 8 & 13 \\
\hline 96 & 11 & 9 & 8 & 8 & 14 \\
\hline 97 & 7 & 6 & 6 & 6 & 8 \\
\hline 98 & 10 & 9 & 7 & 5 & 6 \\
\hline 99 & 8 & 7 & 7 & 7 & 10 \\
\hline 100 & 7 & 6 & 6 & 6 & 9 \\
\hline 101 & 10 & 8 & 7 & 7 & 13 \\
\hline 102 & 13 & 11 & 9 & 9 & 17 \\
\hline 103 & 16 & 13 & 11 & 9 & 24 \\
\hline 104 & 10 & 9 & 8 & 8 & 10 \\
\hline 105 & 11 & 9 & 8 & 8 & 11 \\
\hline 106 & 14 & 11 & 9 & 7 & 12 \\
\hline 107 & 17 & 14 & 11 & 9 & 16 \\
\hline 108 & 20 & 17 & 13 & 11 & 20 \\
\hline 109 & 23 & 19 & 14 & 13 & 27 \\
\hline 110 & 20 & 16 & 12 & 11 & 20 \\
\hline 111 & 17 & 13 & 10 & 9 & 16 \\
\hline 112 & 14 & 11 & 10 & 10 & 15 \\
\hline 113 & 17 & 14 & 11 & 11 & 16 \\
\hline 114 & 14 & 12 & 9 & 9 & 12 \\
\hline 115 & 17 & 14 & 11 & 11 & 13 \\
\hline 116 & 14 & 11 & 9 & 9 & 12 \\
\hline 117 & 11 & 9 & 7 & 7 & 11 \\
\hline 118 & 8 & 6 & 5 & 5 & 6 \\
\hline 119 & 11 & 8 & 7 & 7 & 10 \\
\hline 120 & 14 & 11 & 10 & 10 & 15 \\
\hline
\end{tabular}


TABLE III: COMPARISON BETWEEN N" AND GISN

\begin{tabular}{l|r}
\hline & The number of access structures \\
\hline$N^{\prime \prime}<g_{I S N}$ & 169 \\
$N^{\prime \prime}=g_{I S N}$ & 11 \\
$N^{\prime \prime}>g_{I S N}$ & 0 \\
\hline
\end{tabular}

\section{CONCLUSION}

We have proposed a new secret sharing scheme realizing general access structures. The proposed scheme is perfect and can reduce the number of shares distributed to each participant. We can apply the proposed scheme to the same access structure recursively. The proposed scheme is more efficient than or equal to Benaloh and Leichter's scheme [12] for any access structure. Furthermore, we have shown that the proposed scheme is more efficient than or equal to Ito, Saito and Nishizeki's scheme [11] for all 180 access structures on five participants. We will compare the proposed scheme with the other general secret sharing schemes and evaluate the efficiencies in the follow-up work.

\section{REFERENCES}

[1] A. Shamir, "How to share a secret," Comm. ACM, vol. 22, no. 11, pp. 612-613, 1979

[2] G. Blakley, "Safeguarding cryptographic keys," Proceedings of AFIPS, vol. 48, pp. 313-317, 1979 .

[3] K. Koyama, "Cryptographic key sharing methods for multi-groups and security analysis," Trans. of the IECE, vol. E66, no. 1, pp. 13-20, 1983.

[4] G. Simmons, "How to (really) share a secret," in Proc CRYPTO, vol. 88, pp. 390-448, 1988.

[5] G. Simmons, "Prepositioned shared secret and/or shared control schemes," in Proc. EUROCRYPT, vol. 89, pp. 436-467, 1989.

[6] T. Tassa, "Hierarchical threshold secret sharing," Journal of Cryptology, Vol. 20, pp.237-264, 2007.

[7] Farrás and C. Padró, "Ideal hierarchical secret sharing schemes," IEEE Trans. on IT, vol. 58, no. 5, pp. 3273-3286, 2012.

[8] H. Sun, H. Wang, B. Ku, and J. Pieprzyk, "Decomposition construction for secret sharing schemes with graph access structures in polynomial time," SIAM Journal on Discrete Mathematics, vol. 24, no. 2, pp. 617-638, 2010.

[9] L. Csirmaz, P. Ligeti, and G. Tardos, "Erdös-Pyber theorem for hypergraphs and secret sharing," Graphs and Combinatorics, vol. 31, no. 5, pp. 1335-1346, 2015.

[10] A. Beimel, Y. Mintz, and O. Farrás, "Secret-sharing schemes for very dense graphs," Journal of Cryptology, vol. 29, issue 2, pp. 336-362, 2016.
[11] M. Ito, A. Saito, and T. Nishizeki, "Secret sharing scheme realizing general access structure," in Proc. IEEE Globecom, vol. 87, pp. 99-102, 1987.

[12] J. Benaloh and J. Leichter, "Generalized secret sharing and monotone functions," in Proc. CRYPTO, vol. 88, pp. 27-35, 1988.

[13] K. Tochikubo, "Efficient secret sharing schemes realizing general access structures," IEICE Trans. Fundamentals, vol. E87-A, no. 7, pp. 1788-1797, 2004.

[14] K. Tochikubo, "Efficient secret sharing schemes based on unauthorized subsets," IEICE Trans. Fundamentals, vol. E91-A, no. 10 , pp. 2860-2867, 2008

[15] K. Tochikubo, T. Uyematsu, and R. Matsumoto, "Efficient secret sharing schemes based on authorized subsets," IEICE Trans. Fundamentals, vol. E88-A, no. 1, pp. 322-326, 2005

[16] K. Tochikubo, "New construction methods of secret sharing schemes based on authorized subsets," Journal of Information Processing, vol. 21, no. 4, pp. 590-598, 2013.

[17] D. R. Stinson, Cryptography: Theory and Practice, 3rd edition, CRC Press, 2005

[18] E. D. Karnin, J. W. Greene, and M. E. Hellman, "On secret sharing systems," IEEE Trans. on IT, vol. 29, no. 1, pp. 35-41, 1983.

[19] W. Jackson and K. M. Marin, "Perfect secret sharing schemes on five participants," Designs, Codes and Cryptography, vol. 9, issue 3, pp 267-286, 1996.

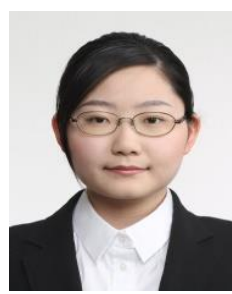

Utako Itoh received her B.E. degree from Nihon University in 2016. Currently, she is a master's course student in the Department of Mathematical Information Engineering, Graduate School of Industrial Technology, Nihon University.

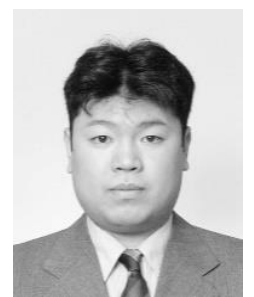

Kouya Tochikubo received his B.S. degree from Tokyo University of Science, his M.S. degree from Japan Advanced Institute of Science and Technology, and his D.E. degree from Tokyo Institute of Technology in 1996, 1998 and 2004, respectively. He joined the Systems Integration Technology Center, Toshiba Corporation in 1998. Currently, he is an associate professor in the Department of Mathematical Information Engineering, College of Industrial Technology, Nihon University. He was a visiting professor at the University of Waterloo from 2012 to 2013. Dr. Tochikubo received the SCIS Paper Award and the Institute of Electronics, Information and Communication Engineers (IEICE) Best Paper Award in 2002 and 2005 respectively. 